\title{
Distribuição espacial dos casos de leishmaniose tegumentar americana no município de Campinas, Estado de São Paulo, no período de 1992 a 2003
}

\author{
Spatial distribution of American tegumentary leishmaniasis cases \\ in Campinas, State of São Paulo, between 1992 and 2003
}

\author{
Jeanette Trigo Nasser ${ }^{1}$, Maria Rita Donalisio ${ }^{2}$ \\ e Cíntia Honório Vasconcelos ${ }^{3}$
}

\begin{abstract}
RESUMO
Trata-se de estudo retrospectivo com componente ecológico que descreve o padrão epidemiológico e a distribuição geográfica da leishmaniose tegumentar americana no município de Campinas, SP de 1992 a 2003. Os locais prováveis de infecção foram georeferenciados por meio de Sistema de Posicionamento Global e espacializados pelo programa Spring 4.01 do Instituto Nacional de Pesquisas Espaciais. Foi aplicado o estimador de intensidade kernel para se obter as regiões com maior frequiência de casos. Identificou-se a ocorrência de 3 surtos (região Leste e Sudoeste) nos anos de 1993/1995 e 2002/2003. Foram estudadas variáveis sócio-demográficas, proximidade do domicílio à mata, tempo de moradia e forma clínica da doença. Embora as características ecológicas e sócio-ambientais das áreas de estudo sejam diferentes, os surtos apresentaram perfil semelhante. A distribuição por sexo, idade e ocupação sugere possível transmissão peridomiciliar. A proximidade dos locais prováveis de infecção das matas foi comum a todas as áreas.
\end{abstract}

Palavras-chaves: Leishmaniose tegumentar americana. Vigilância epidemiológica. Distribuição espacial.

\section{ABSTRACT}

This is a retrospective study with ecological features that describes the epidemiological pattern and geographical distribution of American tegumentary leishmaniasis cases in Campinas, São Paulo, between 1992 and 2003. The probable infection locations were georeferenced by means of Global Position System and spatially described using Spring 4.01 Beta software from the Brazilian National Space Research Institute. A kernel estimator was applied to identify areas of case concentration, three epidemic areas with higher case intensity were found in the municipal area in 1993/1995 and 2002/2003. Socio-demographic (gender, age, occupation, residence time), closeness of domicile to forest, and clinical form of the disease were studied. Although socio-environment characteristics of the areas were different, epidemics profile were similar. Age, sex and occupational distribution suggest peri-domestic transmission. Proximity to forest has being a risk factor.

Key-words: American tegumentary leishmaniasis. Epidemiologic surveillance. Spatial analysis.

A leishmaniose tegumentar americana (LTA) é uma doença causada por protozoário do gênero Leishmania de caráter zoonótico e transmissão vetorial, de ampla distribuição nas Américas ${ }^{8}$. No Brasil, observa-se uma expansão geográfica dessa enfermidade. No período de 1980 a 2004, a LTA apresentou coeficientes de detecção com tendência ao crescimento, que oscilaram entre 3,8 e 22,9 por 100.000 habitantes. Os coeficientes mais elevados ocorreram nos anos de 1994 e 1995 ,

1. Prefeitura Municipal de Campinas, Campinas, SP. 2. Departamento Medicina Preventiva e Social, Faculdade de Ciências Médicas, Universidade de Campinas, Campinas, SP. 3. Centro de Pesquisa René Rachou, Fundação Oswaldo Cruz, Belo Horizonte, MG. Endereço para correspondência: Dra Maria Rita Donalisio. Laboratório de Análises Espaciais de Dados Epidemiológicos/Depto de Medicina Preventiva e Social/ FCM/UNICAMP. Caixa Postal 6111, Cidade Universitária, 13083-970 Campinas, SP. Telefax:55 19 3521-8036

e-mail: donalisi@fcm.unicamp.br

Recebido para publicação em 19/05/2008

Aceito em 09/06/2009 quando atingiram níveis de 22,8 e 22,9 por 100.000 habitantes, respectivamente ${ }^{13}$.

No Estado de São Paulo, nas últimas décadas, o maior registro de casos foi nos anos de 1993 e 1994, com 835 e 843 casos e coeficientes de detecção de 2,6 a 2,5 por 100.000 habitantes, respectivamente ${ }^{10}$.

Em decorrência da grande diversidade de contextos ambientais de transmissão da LTA, as ações de controle são dificultadas. Isso ocorre em razão da existência de diferentes espécies de vetores, reservatórios e agentes etiológicos associados à ação do homem sobre o meio ambiente ${ }^{89}$. As estratégias para o controle devem ser específicas, daí a necessidade de se considerar o padrão epidemiológico regional e o comportamento da transmissão em cada local.

O objetivo deste estudo é analisar a distribuição espacial e o padrão epidemiológico da LTA em Campinas, SP, nos anos de 1992 a 2003. 


\section{MATERIAL E MÉTODOS}

Este é um estudo retrospectivo descritivo dos casos de LTA e abordagem ecológica sobre a ocorrência de LTA no município de Campinas, situado a nordeste do Estado de São Paulo (Figura 1), distante $100 \mathrm{~km}$ da capital paulista e com população de 1.045.706 habitantes. Seu território está inserido em dois grandes domínios geomorfológicos: a depressão periférica e o planalto atlântico. Caracteriza-se como um importante pólo industrial e migratório do estado com grande expansão demográfica nas últimas décadas.

Foi investigada a totalidade $(\mathrm{n}=60)$ dos casos de LTA notificados no período de 1992 a 2003 disponíveis pelo Sistema de Informação sobre Agravos Notificáveis (SINAN), a partir de 1998 e nos anos anteriores obtidos das fichas de investigação epidemiológicas disponibilizados pelo Sistema de Vigilância Epidemiológica municipal e estadual. A pesquisa e identificação de espécies de vetores foram realizadas pela equipe da Superintendência de Controle de Endemias (SUCEN) Campinas, na ocasião da notificação dos casos.

Os locais prováveis de infecção dos 60 casos foram localizados e marcados no campo com auxilio de Sistema de Posicionamento Global (SPG) no Datum Córrego Alegre. A projeção utilizada foi o Sistema de Coordenadas Transversais Universal de Mercator (UTM) ${ }^{2}$.

Após a coleta das coordenadas, os dados foram transferidos para o programa Spring 4.01 Beta do Instituto Nacional de Pesquisas Espaciais (INPE). Utilizou-se o estimador de intensidade kernel que permite a análise do comportamento de primeira ordem de padrões de pontos com o objetivo de determinar a tendência espacial na distribuição dos casos e identificar a existência de algum padrão sistemático particular'. Foi considerado o raio da banda da função kernel de $2 \mathrm{~km}$ para a elaboração das superfície de densidade de casos, suficiente para delimitar localidades com características ecológicas semelhantes e pertencentes ao mesmo ecossistema, possível gerador de transmissão.

A partir destas imagens, foram identificados aglomerados espaciais (mais de 3 casos) e temporais de casos, os quais foram considerados surtos da doença. Para tal definição, comparou-se esta ocorrência com os registros dos últimos 10 anos, quando não foi identificado nenhum caso de leishmaniose tegumentar americana no municípi $0^{113}$. A delimitação temporal dos surtos foi ao redor de 2 anos, considerado-a adequada para se analisar esta doença crônica, com grande período de incubação, demora no diagnóstico e na notificação. A delimitação espacial dos casos foi estabelecida em aproximadamente $2 \mathrm{~km}$, espaço que delineia as localidades e suas conexões na zona rural do município.

Não foi realizada a razão de kernel, tampouco a aplicação de modelos lineares generalizados para a incorporação da distribuição populacional na análise de riscos, devido à dificuldade de se obter a distribuição do contingente populacional no mapa da cidade.

Foi estudado o perfil epidemiológico dos surtos mediante a análise das seguintes variáveis: sociodemográficas (sexo, idade, ocupação, tempo de moradia), proximidade do domicílio à mata, forma clínica, número de lesões e distribuição espacial dos locais prováveis de infecção (LPI). Estes foram definidos como o domicílio do indivíduo na ocasião da infecção ou localidade indicada pela Sucen após investigação de campo. Foi considerada a distância de $200 \mathrm{~m}$ da mata como parâmetro de referência para risco de transmissão. 0 tempo de permanência menor que 2 anos foi considerado marcador de ocupação recente da área. As

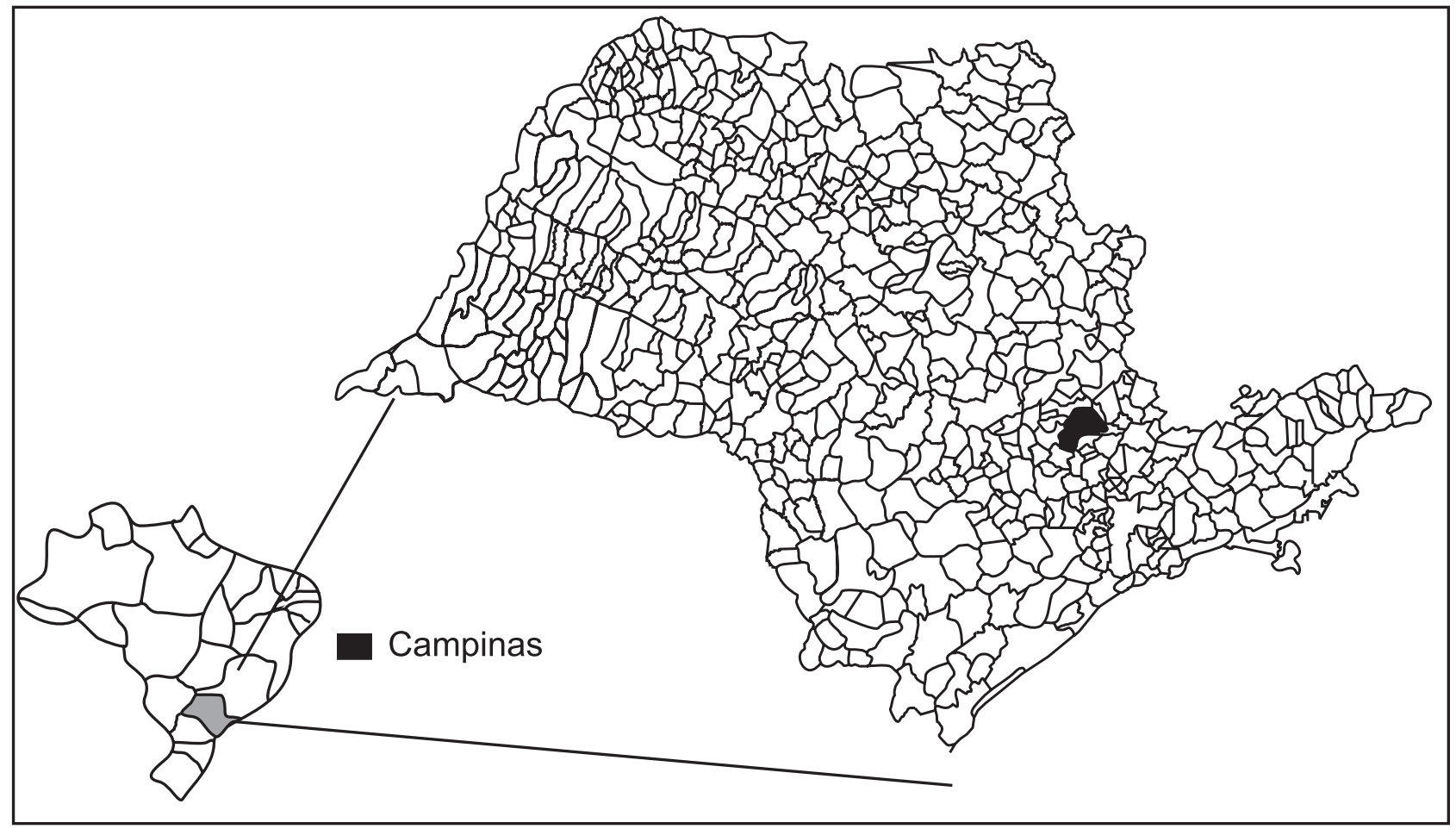

FIGURA 1

Localização da área de estudo, município de Campinas, SP. 
proporções foram comparadas utilizando-se o teste qui quadrado, considerando-se o nível de significância de 5\%.

Para elaboração do banco de dados e análise descritiva foi utilizado o programa Epiinfo versão 6.04. 0 trabalho foi aprovado pelo Comitê de Ética em pesquisa da FCM da Unicamp parecer número 241/2006.

\section{RESULTADOS}

Foram estudados 60 casos que ocorreram durante todo 0 período de estudo. A Figura 2 ilustra a distribuição espacial dos locais prováveis de infecção da LTA ocorridos em Campinas no período de 1992 a 2003. Nota-se que o número de pontos observados é inferior a 60 por haver em alguns locais a representação de mais de um caso. A grande maioria, 54 (90\%) apresentou como LPI, a própria residência situada sempre próxima ou dentro da mata; 95,6\% estavam situadas a menos de 200m de uma mata.

A Figura 2 mostra as áreas de maior concentração dos casos na zona rural margeando a área urbana em dois extremos da cidade. Observam-se duas áreas que se sobressaem em relação às demais, uma a Leste e outra a Sudoeste. A Figura 3 apresenta a distribuição temporal dos casos, concentrados nos anos de 1993/1995 (46 casos) na região Leste (29) e Sudoeste (12) e em 2002/2003 ( 5 casos) na região Sudoeste.

A idade média de todos os indivíduos acometidos no período foi de 26,6, mediana 23 e desvio padrão 18,9 anos. Na análise do tempo de permanência no local provável de infecção, observou-se que a média foi de 6,7 anos, o desvio padrão de 7,1 e mediana de 4,5 anos. Em 11 (18,3\%) casos, o tempo de permanência foi menor que 2 anos.

Quanto ao número de lesões, 38 (63,3\%) indivíduos apresentaram lesão única, 5 (8,3\%) apresentaram duas lesões e $11(18 \%)$ apresentaram três ou mais lesões. Em relação ao grau de escolaridade, apenas 12 (20\%) indivíduos possuíam essa informação registrada, o que inviabilizou a análise dessa variável.

0 padrão sócio-demográfico e epidemiológico dos surtos de ITA ocorridos em 1993/1994 é semelhante, sem predomínio da doença entre os sexos, com acometimento de todas as faixas etárias e grande ocorrência de casos entre menores, donas de casa e agricultores. Quanto à forma clínica só se observou a cutânea, e a respeito do tempo de permanência na localidade, registrou-se permanência maior na região Sudoeste. Embora houvesse 6 informações ignoradas na região Sudoeste dificultando a comparação, na região Leste, os LPI localizavam-se mais próximos à mata (80\%). (Tabela 1).

Entre os cinco casos notificados na região Sudoeste em 2002 e 2003, três ocorreram em mulheres, três em menores de 14 anos, todos da forma cutânea e em moradores a menos de $200 \mathrm{~m}$ da mata, sendo apenas 1 com menos de 2 anos de permanência na área.

Em investigação dos LPI realizada pela equipe da Sucen, registrou-se predomínio da Lutzomyia intermedia nas margens da mata e da Lutzomyia whitmani na zona periurbana da região Leste. Nos surtos da região Sudoeste em 2003, registraram-se sete espécies diferentes de flebotomíneos, sendo a mais abundante Lutzomyia pessoai (45\%) e a menos Lutzomyia intermedia (1,9\%).

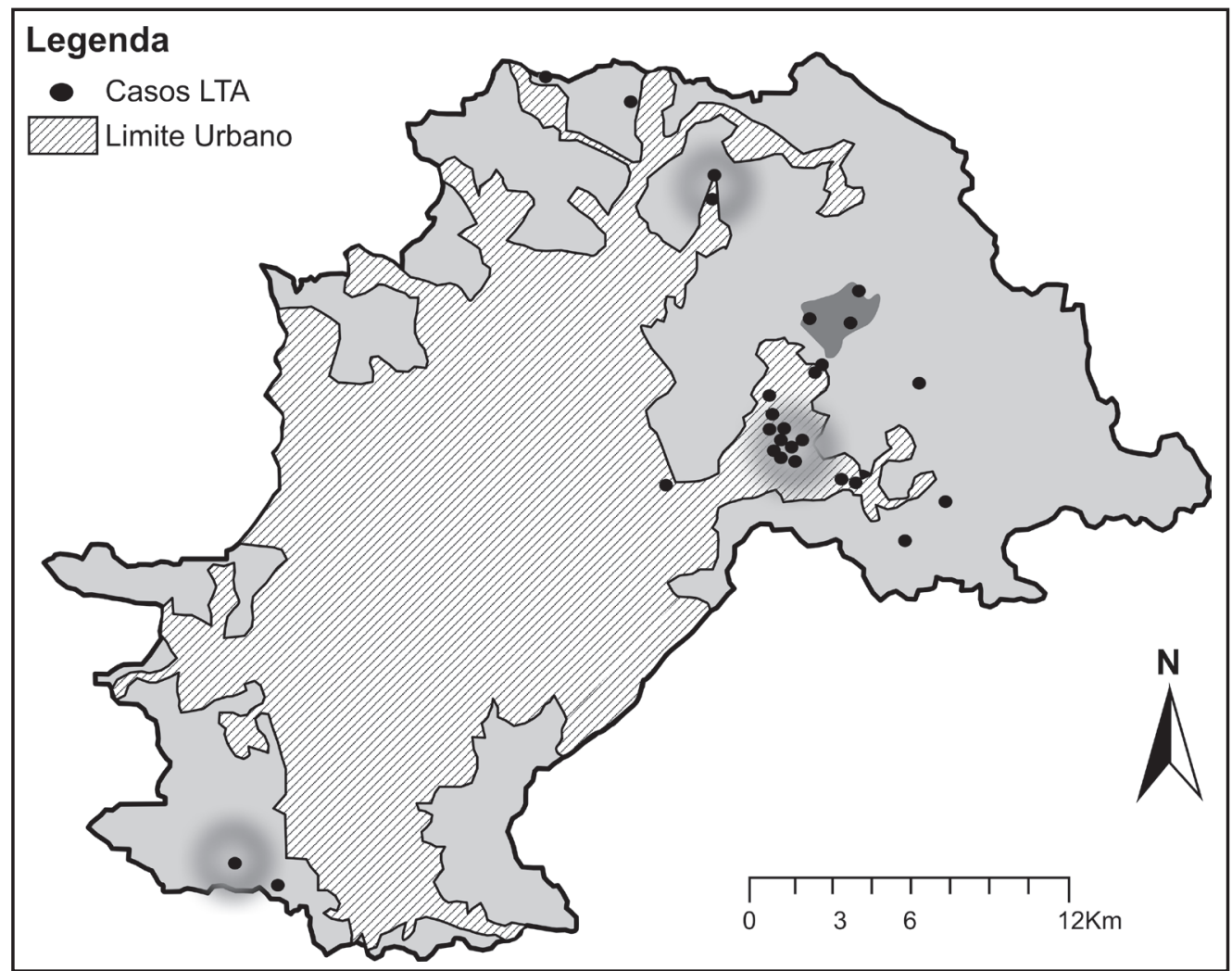

FIGURA 2

Mapa dos locais prováveis de infecção de leishmaniose tegumentar americana, estimador de intensidade kernel e área urbana (2003), em Campinas, SP, no período de 1992 a 2003. 
TABELA 1

Características sócio-demográficas e epidemiológicas dos casos nos surtos de leishmaniose tegumentar no município de Campinas, 1992 a 1995.

\begin{tabular}{|c|c|c|c|c|c|}
\hline \multirow[b]{3}{*}{ Variáveis } & \multirow{2}{*}{\multicolumn{2}{|c|}{$\begin{array}{c}\text { Leste } \\
1993 \text { a } 1995\end{array}$}} & \multicolumn{2}{|c|}{ Sudoeste } & \multirow{3}{*}{$\mathrm{p}$} \\
\hline & & & & & \\
\hline & $\mathrm{n}^{0}$ & $\%$ & $\mathrm{n}^{\mathrm{o}}$ & $\%$ & \\
\hline Sexo & & & & & 0,48 \\
\hline masculino & 14 & 48,3 & 8 & 66,7 & \\
\hline feminino & 15 & 51,7 & 4 & 33,3 & \\
\hline Faixa etária (anos) & & & & & 0,56 \\
\hline$\leq 14$ & 11 & 37,9 & 6 & 50,0 & \\
\hline 15 a 29 & 9 & 31,1 & 4 & 33,3 & \\
\hline 30 a 59 & 8 & 27,6 & 2 & 16,7 & \\
\hline$\geq 60$ & 1 & 3,4 & 0 & 0,0 & \\
\hline Ocupação & & & & & - \\
\hline estudante & 7 & 24,2 & 0 & 0,0 & \\
\hline do lar & 8 & 27,6 & 0 & 0,0 & \\
\hline agricultor & 5 & 17,2 & 3 & 25,0 & \\
\hline outras ${ }^{* * *}$ & 5 & 17,2 & 1 & 8,3 & \\
\hline menor & 4 & 13,8 & 6 & 50,0 & \\
\hline ignorada & 0 & 0,0 & 2 & 16,7 & \\
\hline Forma clínica & & & & & $0,29 *$ \\
\hline cutânea & 29 & 100,0 & 11 & 91,7 & \\
\hline ignorada & 0 & 0,0 & 1 & 8,3 & \\
\hline Distância domicílio-mata (m)**** & & & & & $0,05^{*}$ \\
\hline$\leq 200$ & 24 & 82,8 & 6 & 50,0 & \\
\hline$>200$ & 0 & 0,0 & 0 & 0,0 & \\
\hline ignorada & 5 & 17,2 & 6 & 50,0 & \\
\hline Tempo de permanência (anos) $)^{* * * * *}$ & & & & & 0,46 \\
\hline$\leq 2$ & 6 & 20,6 & 1 & 8,3 & \\
\hline 2 a 5 & 9 & 31,1 & 2 & 16,7 & \\
\hline$\geq 6$ & 12 & 41,4 & 8 & 66,7 & \\
\hline ignorado & 2 & 6,9 & 1 & 8,3 & \\
\hline
\end{tabular}

*Aplicação do Teste qui quadrado e teste exato de fisher bicaudal

** Outras profissões: comerciante, metalúrgico, engenheiro, pedreiro, motorista.

**** Comparação entre $\leq 200 \mathrm{~m}$ e ignorados com

*****os ignorados não incluídos na análise estatística.

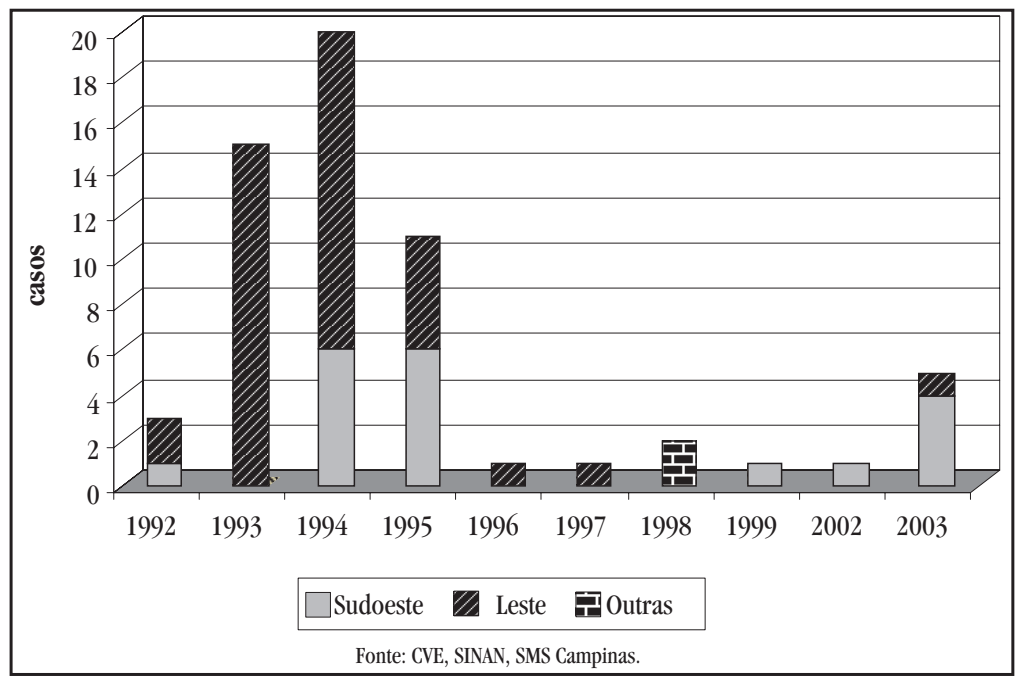

FIGURA 3

Distribuição anual de casos de leishmaniose tegumentar americana em Campinas, SP, período de 1984 a 2003. 


\section{DISCUSSÃo}

A distribuição dos casos de LTA não foi homogênea ao longo dos anos. Observam-se dois períodos em que se concentraram a maioria dos casos, 1993 a 1995 e 2002 a 2003. Estes casos ocorreram em áreas rurais bem delimitadas na região Leste e Sudoeste do município. Foram considerados surtos da doença, devido ao registro não usual de casos em áreas delimitadas no tempo e espaço, embora este seja um comportamento esperado da doença na região, isto é, a ocorrências de casos em pequenos surtos seguidos por períodos de silêncio epidemiológico ${ }^{11}$.

0 mapa do estimador de intensidade de kernel identificou aglomerados no espaço (Figura 2) e o gráfico da distribuição temporal da ITA (Figura 3) apontou anos de maior detecção de casos. Estes dados não permitem estimar riscos de ocorrência da doença ponderados pela população das áreas de estudo, apenas identificam locais e períodos de transmissão mais intensa, comparando-se com registros pregressos da doença. No caso da LTA a delimitação de áreas rurais e semi-rurais onde se concentraram os casos e o estudo de características ambientais e ecológicas destes locais podem ser marcadores de interesse para a compreensão da dinâmica de transmissão, ainda pouco desvendada ${ }^{4} 812$. Optou-se por georreferenciar os pontos (LPI), os quais podem trazer informações mais precisas sobre a área de risco, pois referem-se a localidades (sítios, fazendas, bairros) onde habitam indivíduos com infecção e apresentam condições ecológicas favoráveis à ocorrência da doença ${ }^{11}$. Assim, foram analisados os pontos e não taxas de incidências por bairros, setores censitários ou outros recortes da área urbana, procurando delimitar regiões de maior transmissão.

Corte e cols descreveram o surto da doença em parte da região Leste (Sousas e Joaquim Egídeo) associando o surgimento de casos à expansão urbana com desmatamento e preservação de pequenas áreas circunscritas de matas residuais, ciliares e resultantes de reflorestamento 5 . Após a consolidação imobiliária na região notificou-se um silêncio epidemiológico embora as matas continuem preservadas. A área urbana na região Leste, na qual o surto de ITA está localizado foi decorrente da expansão urbana no início da década de 90, um dos contextos que explicaram a concentração de casos naquela região em 1992 e 1993.

No período de 1992 a 1995, também ocorreu surto (13 casos) na área Sudoeste, fato que se repetiria após uma década na mesma localidade; porém, com menor intensidade (5 casos). Naquele período, em ambas as áreas, o contato com a mata ciliar e a localização dos LPI próximos a áreas de mata preservada, foi registrada em grande parte dos casos.

Em 2002/2003, não houve recidivas na região Sudoeste e sim novos susceptíveis, inclusive parentes dos indivíduos acometidos na década anterior. Esse comportamento cíclico da doença e o por quê da não repetição dos casos na área Leste ressaltam a importância de estudos que analisem variáveis ecológicas e ambientais, destacando-se a forma de ocupação do espaço rural que pode ter relevância na transmissão da doença.

A área Leste caracteriza-se por ser uma região com grande número de condomínios residenciais de médio a alto padrão socioeconômico na proximidade da bacia do rio Atibaia. Após a fase de construção e alterações na paisagem local, o panorama imobiliário da região se consolidou com moradores habitando condomínios sem contato direto com a mata ciliar. As manchas de mata nativa na região Leste ficam mais distantes que na região Sudoeste, inserida na bacia do rio Capivari, e que se caracteriza por pequenas propriedades rurais, onde predominam atividades agrícolas e criação de animais.

Por outro lado, ao se analisar a distribuição dos 60 casos notificados durante todo o período, verificou-se padrão semelhante ao observado em outros municípios do Estado e do país, isto é, o acometimento de ambos os sexos e de todas as faixas etárias, sugere que a transmissão pode estar ocorrendo no ambiente peri ou intradomiciliar ${ }^{3710}$ A proximidade do domicílio às matas é um fator de risco observado na maioria dos casos estudados, dificultando precisar se a transmissão se deu no peri ou no intradomicílio ou no interior das matas.

$\mathrm{Na}$ maior parte dos casos, a forma clínica foi a cutânea (96,7\%) e com lesão única (63,3\%), como mostram outros estudos na região Sudeste do Brasil ${ }^{14}$.

Verificou-se que o tempo de permanência médio no local provável de infecção foi de 6,7 anos sendo que apenas 8,3\% dos indivíduos tinham permanência maior que 15 anos, o que sugere tratar-se de transmissão recente nas regiões dos surtos. Estes achados estão de acordo com estudo feito por Rangel (2004) abrangendo 14 municípios da região de Campinas ${ }^{11}$. 0 tempo de permanência no local provável de infecção é uma variável mais adequada para se analisar o risco de adquirir LTA, quando se compara com a idade, em virtude da frequente mobilidade dos indivíduos no país ${ }^{912}$.

Há que se considerar a dificuldade em se trabalhar com dados secundários de leishmaniose tegumentar americana, os quais apresentam frequente subnotificação de casos, mau preenchimento das fichas epidemiológicas e inexatidão de informações referentes a esta doença crônica com grande período de incubação e evolução.

Com relação aos vetores, as coletas foram realizadas de forma pontual, relacionando espécies de flebotomíneos presentes no local provável de infecção. As espécies foram semelhantes às encontradas por outros autores no Estado ${ }^{4} 611$. Estudos longitudinais sobre o comportamento de espécies de vetores frente a mudanças ambientais e a sazonalidade podem contribuir para uma melhor compreensão da doença na região. Além disso, ressalta-se a importância de desenvolver estudos dos reservatórios silvestres e domésticos da LTA.

Concluindo-se, o estudo de surtos de LTA pode indicar tendências do comportamento da doença em determinado contexto ecológico. Os surtos analisados neste estudo apresentaram características semelhantes embora tenham ocorrido em regiões distintas da cidade e com condições ambientais e sócioeconômicas diversas. 0 contexto ambiental, social e fatores como o uso e ocupação do solo podem estar envolvidos na transmissão da doença, explicando a não recorrência de casos na região Leste. Estudos mais aprofundados poderiam esclarecer estas hipóteses. 


\section{REFERÊNCIAS}

1. Bailey T, Gatrell AC. An Interactive spatial data analysis. Longman Scientific and Technical Publisher, London, 1995.

2. Burrough PA. Principles of geographic information systems for land resources assessment. Publisher Claredon Press, Oxford, 1986.

3. Castro EA, Soccol VT, Membrive NE. Estudo das características epidemiológicas e clínicas de 332 casos de leishmaniose tegumentar notificados na região norte do estado do Paraná de 1993 a 1998. Revista da Sociedade Brasileira de Medicina Tropical 35:445-452, 2002.

4. Condino ML, Azevedo CC, Toia RM. Aspectos epidemiológicos da leishmaniose Tegumentar americana no município de Ubatuba, Litoral Norte, São Paulo - Brasil, 1993-2003. Boletim Epidemiológico Paulista1:6-7, 2004.

5. Corte AA, Ferreira MC, Felipe PAN, Pignatti MG, Rangel 0, Souza SS, Nozawa MR. Aspectos ecoepidemiológicos da LTA no município de Campinas. Cadernos de Saúde Pública 12:465-472, 1996.

6. Fundação Nacional de Saúde. Manual de Controle da Leishmaniose Tegumentar Americana,. Assessoria de Comunicação e Educação em Saúde-NED/ASCOM/ FUNASA, Ministério da Saúde, Brasília/DF, 2000.

7. Gomes AC. Sandfly vectorial ecology in the State of São Paulo. Memórias do Instituto Osvaldo Cruz 89: 457-460, 1994
8. Gomes AC, Camargo Neves VLF. Estratégias e perspectivas de controle da leihmanioses tegumentar americana no Estado de São Paulo. Revista da Sociedade Brasileira de Medicina Tropical 31:553-558, 1998.

9. Gontijo B, Carvalho MLR. Leishmaniose Tegumentar Americana. Revista da Sociedade Brasileira de Medicina Tropical 36:71-80, 2003.

10. Katz G. Epidemiologia da Leishmaniose Tegumentar Americana no estado de São Paulo período de 1986 a 1995. Dissertação de Mestrado, Universidade de São Paulo, São Paulo, 1997.

11. Rangel 0. Estudo da expansão das áreas geográficas de transmissão de leishmaniose tegumentar americana (LTA) na região de Campinas - Estado de São Paulo-Brasil. Tese de Doutorado, Universidade Estadual de Campinas, Campinas, 2004

12. Sabroza PC. O domicílio como fator de risco na leishmaniose tegumentar americana - Estudo epidemiológico em Jacarepaguá, município do Rio de Janeiro. Dissertação de Mestrado. Escola Nacional de Saúde Pública, Rio de Janeiro, 1981.

13. Secretaria de Vigilância em Saúde. Disponível em: http://portal.saude.gov.br/ portal/svs Acesso em 15/07/2006.

14. Sessa PA, Barros CG, Mattos EAVRD, Alencar JTA, Delmaestro D. Distribuição geográfica da leishmaniose tegumentar americana no estado do Espírito Santo - Brasil. Revista da Sociedade Brasileira de Medicina Tropical 18:237-241, 1985 